\title{
NOVEL ADAPTIVE FILTER (NAF) FOR IMPULSE NOISE SUPPRESSION FROM DIGITAL IMAGES
}

\author{
Geeta Hanji ${ }^{1}$ and M.V.Latte ${ }^{2}$ \\ ${ }^{1}$ PDA Engineering College, Gulbarga, Karnataka, India. \\ ${ }^{2}$ JSSIT,Bangalore,Karnataka,India.
}

\begin{abstract}
In general, it is known that an adaptive filter adjusts its parameters iteratively such as size of the working window, decision threshold values used in two stage detection-estimation based switching filters, number of iterations etc. It is also known that nonlinear filters such as median filters and its several variants are popularly known for their ability in dealing with the unknown circumstances. In this paper an efficient and simple adaptive nonlinear filtering scheme is presented to eliminate the impulse noise from the digital images with an impulsive noise detection and reduction scheme based on adaptive nonlinear filter techniques. The proposed scheme employs image statistics based dynamically varying working window and an adaptive threshold for noise detection with a Noise Exclusive Median (NEM) based restoration. The intensity value of the Noise Exclusive Median (NEM) is derived from the processed pixels in local neighborhood of a dynamically adaptive window. In the proposed scheme use of an adaptive threshold value derived from the noisy image statistics returns more precise results for the noisy pixel detection. The proposed scheme is simple and can be implemented as either a single pass or a multi-pass with a maximum of three iterations with a simple stopping criterion. The goodness of the proposed scheme is evaluated with respect to the qualitative and quantitative measures obtained by MATLAB simulations with standard images added with impulsive noise of varying densities. From the comparative analysis it is evident that the proposed scheme out performs the state-of-art schemes, preferably in cases of high-density impulse noise.
\end{abstract}

\section{KEYWORDS}

Nonlinear filter, median based filter, Noise exclusive median (NEM), adaptive window, local neighborhood, impulse noise, Peak-Signal-to-Noise Ratio (PSNR)

\section{INTRODUCTION}

One of the challenges researchers in the field of image processing facing today is noise suppression from the images with detail preservation such as edges. In digital images, impulsive noise is more predominant and is added with the images during acquisition, storage, and transmission [1]. Two types of impulse noise are : fixed valued (salt and pepper) or random valued. In the case of salt and pepper noise the noise affected pixels assume the maximum[or '255'] or minimum[ or' 0'] gray levels, whereas in the case of random valued impulse noise the noisy pixels may be any value within the dynamic range of minimum or maximum (i.e. ' 0 ' to ' 255 ' range) gray levels [2]. The prime objective and the main thrust in any scheme of DOI: $10.5121 / \mathrm{ijbb} .2014 .4401$ 
nonlinear filtering are to suppress the impulsive noise while preserving the image details in the output restored images [2,3]. The existing impulse noise removal schemes are classified into two categories, namely i) filtering without detection and ii) filtering with detection-estimation (or detection prior to filtering) scheme. Under the first category falls the nonlinear standard median filter (SMF) [3].Goodness of this filter is its simplicity of implementation and the computational efficacy. But it's performance degrades with the images containing high density of impulsive noise. More over all the filters which fall under the first category perform filtering operation uniformly on all pixels of an image irrespective of their status of corruption and as a result the restored image suffers from loss of precious details such as edges and other fine details, whereas in the second category of filters a pixel under consideration (i.e. a reference pixel) is subjected to detection to know whether it is corrupted or not prior to filtering [4]. Thus in these filters, filtering operation is applied only on the corrupted pixels and thus the healthy pixels are maintained un-altered. Much superior performance is realizable from these selective filtering schemes in restoring the noisy images.

Few other schemes which perform very well in low to high density impulse noise are minimum-maximum exclusive mean (MMEM) [5],Progressive switching median filter (PSMF)[6].In MMEM filter the mean of all the remaining pixels after excluding the minimum and the maximum intensity pixel values from the current working window is computed and is used to replace the corrupted central pixel. Simulation results obtained for this filter indicate that the restoration performance is good and quite acceptable even in the presence of the high impulse noise such as (60\%).In [6] detection and removal of impulse noise can be achieved in two separate stages, i.e. impulse detector is applied in the first stage and in the second stage, the noise filter is applied progressively in iterative fashion. Impulse pixels located in the middle of large noise blotches can also be properly detected and filtered by PSMF[6]. Nevertheless, in case of very high noise density situations its performance degrades.

For discriminating 'bad' and 'healthy' pixels and to restore their actual values Tri state median Filter (TSMF) [7] can be used. In this method, noisy pixels are detected by an impulse detector, which takes the outputs from SM and CWM filters and compares them with the original or centre pixel value in order to make a tri-state decision. The switching logic is controlled by the threshold and the output of TSM filter. At low noise densities performance of TSMF is good, but conditions of higher noise densities yield poor performance.

Another novel filter with a three-level hierarchical soft-switching noise detection process is suggested in a noise adaptive soft switching median (NASSMF) filter [8].Based on the fuzzy set concept, pixels are classified in to uncorrupted pixel, isolated impulse noise, non-isolated impulse noise or image object's edge pixel. It uses "No filtering" (or identity filter), "Standard Median (SM) Filter" or a "Fuzzy Weighted Median (FWM) Filter", the three level switching mechanisms and attempts to effectively remove impulse noise from $10 \%$ to $70 \%$ noise density.

Work presented in [9] proposes a Modified Decision Based Unsymmetrical Trimmed Median Filter (MDBUTMF) that attempts to suppress noise to a greater extent with detail preservation. An improved decision-based algorithm (IDBA) [10] to suppress high density impulse noise is proposed. Using a small and fixed window size of $3 \times 3$, the decision-based algorithm (DBA) replaces the corrupted pixels either by the median pixel or neighbourhood pixels. With the lower processing time which is the main attractive of this filter promising results are obtained, but the degradation in the visual quality of the image with the increased noise density is the main limitation of this technique. 
Fast and efficient decision-based algorithm [11] is proposed to overcome the above stated problem. Results obtained with this filter present better visual appearance for impulse noise corrupted images with good edge preservation capability.

In the design of several, two-stage decision based filters, the threshold value used for decisionmaking is obtained based on empirical means and it remains fixed throughout the detection process. Several random trials are conducted to arrive at a suitable decision threshold $[12,13]$ values.

The problem with the fixed value decision threshold is with reference to the inability of the detector to deal with the images corrupted with low to heavy noise.

Another decision-based median filtering algorithm proposed in [14] attempts to restore the impulse noise corrupted pixels with the median of the pixels scanned in four directions. In [15] an attempt is made to identify the type of noise present the image as additive, multiplicative or impulsive based on the analysis of local histograms. The filter scheme suppresses the detected impulsive noise by employing adaptive nonlinear filtering technique which consists of a process of adaptive noise identification of noisy pixels and filtering by adaptive nonlinear filter.

Method proposed [16] uses a new adaptive noise identification and adaptive nonlinear filtering algorithm to detect and remove the impulsive noise in such a way that the important information of image should be preserved.

The proposed Detail Preserving Fast Median Filtering (DPFMF) [17] scheme is more effective in eliminating fixed value impulse noise while preserving the image details. The filter replaces a corrupted pixel by the fast median filter output or by the value computed based on a unique criterion applied to the processed neighboring pixel values. The uncorrupted pixels are left undisturbed. Simulation studies show that the proposed filter can eliminate impulse noise of densities up to $80 \%$ while preserving the edges and fine details satisfactorily.Work presented in [18] explains an effective filter design based on the average absolute value of four convolutions obtained by one-dimensional Laplacian operators. An effective removal of the impulse noise with a wide range of noise density is achieved to produce better results for images corrupted with noise density as high as $90 \%$.

In [19] the proposed Boundary Discriminative Noise Detection (BDND) scheme is adaptive window technique and performs very effective compared to $[15,16,17,18]$ scheme and also outperforms all the previously proposed median-based filters. The proposed Enhanced BDND [EDND][20] makes use of a faster sorting algorithm both in noise detection and adaptive filtering stages for the restoration of image and video sequences contaminated by impulse noise. The performance of the proposed EBDND is demonstrated through computer simulations in comparison with the BDND method.

Work presented in [21] attempts at obtaining an integrated and consecutive original image from noisy image by a directional correlation-dependent filtering technique by removing salt and pepper noise from the corrupted images. The proposed algorithm can preserve edges very well while removing impulse noise and is very suitable to be applied to many real-time applications.

A Novel Median Filter (NMF) presented in [22] addresses the task of impulsive noise cleaning from the noisy grey scale images and aims at effectively suppressing the impulse noise while preserving the edge information intact. Proposed scheme is simple and yields better denoising performance with better detail preserving up to $60-70 \%$ noise densities, but fails to perform well at higher noise situations. 
Many Filter schemes explained above are 2-stage detection-estimation based approaches and use threshold based identification of noisy pixels. However getting an appropriate threshold value is treated as a cumbersome and trivial process by many researchers. From the above mentioned literature survey, it can be concluded that the Detection-Estimation based filters that derive the decision threshold based on the image statistics are hoped to perform better.

From the above-mentioned literature survey, it is observed that several schemes use window of fixed size to filter the corrupted pixels. In high density impulse noise conditions, a larger working window is needed, whereas in low density noise cases smaller size window is the requirement. Thus to suppress the noisy pixels affected with low to high-density impulse noise with better detail preservation, the above-mentioned filter schemes fail. In general we can conclude saying that the conditions of higher noise densities require working window of larger size for better noise removal which results in less correlation between noise affected pixel values and adaptive thresholds (derived based on the image statistics) in decision making in two stage detection-estimation based filters.

In this research paper, a new Novel Adaptive Filter (or a Noise exclusive median based Adaptive Filter (NemAF) is presented for the suppression of salt and pepper impulse noise. The novelty of the proposal is it's dynamic characteristics that decide the window size for the test pixel before filtering and its adaptive computation capability of deriving an appropriate decision threshold. The exhaustive simulations along with the comparative analysis with the existing schemes in low to high-density impulse noise conditions illustrate the high performance of the proposed scheme.

Organization of the paper is as follows : Model of impulse noise is described in section 2, Section 3 explains the details of the proposed scheme. MATLAB simulation results along with the comparative analysis are discussed in section 4. Finally, section 5 provides the concluding remarks and the scope for future.

\section{NOISE MODEL}

Two classes of impulsive noise that interfere with the images during acquisition, transit and storage are salt-and-pepper noise (SPN) and random-valued impulse noise (RVIN). An image containing impulsive noise [4] can be described as follows:

$$
p(i, j)=\left\{\begin{array}{c}
n(i, j) \text { with probability } r \\
q(i, j) \text { with probability } 1-r
\end{array}\right.
$$

where 'p(i,j)' denotes a noisy image pixel, ' $q(i, j)$ ' denotes a noise free image pixel and $n(i, j$ ) denotes a noisy impulse at the location (i,j).In salt-and-pepper noise, noisy pixels take either minimal or maximal values i.e. $n(i, j) €[$ Pmin , Pmax ]where 'Pmin' and 'Pmax' denote the lowest and the highest pixel intensity values ' 0 ' and ' 255 ' respectively. In random valued impulse noise (RVIN) situations, pixel grey level values range from ' 0 ' to ' 255 ' within the dynamic range. Suppression of such noise is more difficult than the fixed-valued impulse noise because of the differences in gray levels between a noisy pixel and its noise-free neighbors. Work presented in this paper considers only salt and pepper noise which is represented in fig (1) below: 


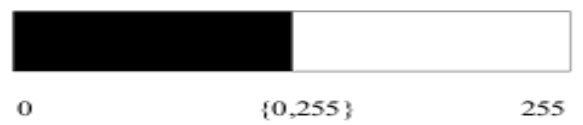

Figure 1. Representation of Salt \& Pepper noise with $\mathrm{P}_{\mathrm{i}, \mathrm{j}} € \mathrm{P}_{\min }$ and $\mathrm{P}_{\max }$

\section{PROPOSED SCHEME}

Novel Median Filter (NAF) scheme consists of five stages, namely, impulse noise detection, adaptive window size selection, adaptive threshold selection, and the selection of the number of iterations and noisy pixel restoration. Description of each stage is given below:

\subsection{Salt and Pepper Impulse noise detection}

Main theme of detection of a noisy pixel involves comparison of its luminance value with a value ' 0 ' or ' 255 ' and counting the number of uncorrupted neighbors to validate whether the required condition is met or not. Based on these findings, decision strategy is framed as below : Declare the reference (or test) pixel $\mathrm{P}(\mathrm{i}, \mathrm{j})$ as noisy iff its luminance value is either a ' 0 ' or ' 255 ' and if it has ' $W$ ' or more number of non-noisy pixels where $W \geq T / 3$, with $T=$ Total number of pixels in the working window.

\subsection{Adaptive Window Size Selection}

Initially, we start with a $3 \mathrm{X} 3$ working window. In the case if the number of non-corrupted pixels in the working is window is insufficient, then the next higher size of the window is sought by the proposed scheme and the process is repeated till the window size reaches to a predefined maximum size of 9X9. Adaptive window serves the need of addressing the images corrupted with the low-to-heavy impulsive noise density.

\subsection{Adaptive Threshold Selection}

Proposed filter scheme identifies the impulse noise based on adaptive threshold whose value is determined from each noisy image to obtain results that are more promising. A new method of deriving an adaptive decision threshold is based on detecting the 'unhealthy' and 'healthy' pixels as presented below in which the restoration algorithm attempts to restore the pixel intensities of only unhealthy pixels. Firstly, initial global thresholds are set based on the following observations:

a) Digital images affected with salt and pepper noise, the presence of salt noise [pixel intensity of ' 0 ' is less observable than pepper noise [pixel intensity of '255'] [18], in the regions near maximum brightness and vice-versa.

b) The loss of sharp image details like edge, boundary etc. is less noticeable than the loss of fine details such as texture etc. in the brighter areas of noise-affected images.

The above mentioned two important observations motivated us to treat the salt and pepper noise components of the added salt and pepper noise from the digital images with two different thresholds 'Ts' an 'Tp'. Initially thresholds 'T1' and 'T2' of twice the mid brightness values are set. Depending on the variations in the mean brightness of the local neighborhoods, threshold values for either salt or pepper noise components increase or decrease. For example, 'Ts' reduces to permit for more salt noise suppression whereas ' $\mathrm{Tp}$ ' increases to permit for more pepper noise 
removal. In the proposed work 'Ts' and 'Tp' are set as follows:

$$
\begin{aligned}
& T_{z}=T_{1} \frac{l(\max )^{2}-l(i, j)}{l(\max )} \\
& T_{p}=T_{2} \frac{l(i, j)}{l[\max )}
\end{aligned}
$$

where $I(i, j)$ is a mean grey level intensity values of the local neighborhood.

Simulations performed on several images corrupted with salt and pepper noise with the initially set thresholds enabled us to conclude that the optimum thresholds required to obtain a better balance between the impulse noise removal and detail preservation are 'one third' of the

maximum grey value, i.e. $\frac{l(\max x)}{a}$. Hence $\mathrm{T} 1=\mathrm{T}_{2}=\frac{l(\max 2)}{a}$ and $\mathrm{I}(\max )=255$ are chosen. Thus,

$$
\begin{aligned}
& T_{3}=\frac{w E-r(h)}{3} \\
& T_{p}=\frac{2(i, j)}{3}
\end{aligned}
$$

where $0 \leq \mathrm{I}(\mathrm{i}, \mathrm{j}) \leq 255$.

\subsection{Selection of Number of Iterations}

Recursive implementation of the proposed filter algorithm helps the de-noised image to minimize to the original (or root) image because, the recursive version of the basic filtering is an optimization operation so that the filter output is always defined by the minimum of a Mean Square Error (MSE) cost function of the output state of the filter [18]. In the Proposed NAF algorithm the procedure used for the recursive filtering can be expressed as below:

$$
o(i, j)=\sum_{p=1}^{q-1} o_{p}(i, j)
$$

where, $O_{p}(i, j)$ for all the values from $p=1$ to $q-1$ are the outputs of previous stage filtering. This approach ensures that the output image converges to the root image within a maximum of four iterations.

\subsection{Noise Filtering}

In order to restore the faulty pixels, the proposed algorithm uses the Noise Exclusive Median (NEM) which is computed as the median of already processed pixels from the current window. In case of a $3 \mathrm{X} 3$ working window, 'NEM' is simply the mean of the second and third pixel intensities in the sorted array. Once the 'NEM' is computed, the restoration of the noisy pixel to obtain an estimate of the original pixel ' $\mathrm{O}(\mathrm{i}, \mathrm{j})$ ' is performed as follows:

$$
O(i, j)=\left[\begin{array}{c}
\left.I(i, j), \mid N E M(i, j)-T_{p}\right]<I(i, j)<\left[N E M(i, j)+T_{2}\right] \\
\text { NEM(i,j), otherwise. }
\end{array}\right.
$$




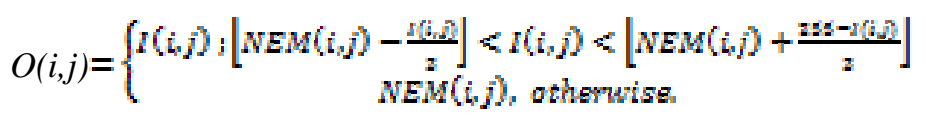

or

$$
O(\mathrm{i}, j)=\left\{\begin{array}{cc}
{[i \mathrm{i}, j), \quad T_{\mathrm{L}}} & <I(\mathrm{i}, j]<T_{n} \\
N E M(, j), \text { otherwise }
\end{array}\right.
$$

where $T^{t} T_{2}$ and ${ }^{n} T_{H}^{t} \quad$ are lower and higher decision thresholds.

Adaptive 2-stage novel algorithm (NAF) proposed in this paper is a detection-estimation based approach in which the novel adaptive threshold based noise detection is followed by the application of NEMF scheme only to the corrupted pixels. The details of the algorithm are given below:

To start with, working window of size $3 \mathrm{X} 3$ is considered. If the total number of non-noisy (healthy) neighbors, excluding already processed pixels is greater or equals to 'three' (or in general one third $(1 / 3)^{\text {rd }}$ of the total number of pixels) then the window size is not altered. Otherwise the working size is incremented to the next higher value until the required condition is met and the window size reaches to a predefined maximum value. Once the suitable window size is chosen, the grey intensity value of the test pixel in the working window is tested to see if it lies between the pre-determined thresholds ' $\mathrm{T}_{\mathrm{L}}$ ' or ' $\mathrm{T}_{\mathrm{H}}$ '. If the test result is 'yes' then it is declared as 'healthy pixel' and is undisturbed. Otherwise, its value is restored with the NEMF output.

\section{RESULTS AND DISCUSSION}

Performance of the proposed NAF scheme is validated with the MATLAB simulation performed on synthetic images like Cameraman, Lena etc. The test images are corrupted with $10 \%$ to $90 \%$ noise densities. At various noise densities the size of the window is made variable. Qualitative as well as quantitative results are obtained and the evaluations have been made on several restored images. The objective measures used are:

1) Peak Signal to Noise Ratio (PSNR)

$$
P S N R=10 \log _{n} \frac{255^{2}}{M S E}
$$

where, Mean Square Error (MSE) for an image of size MXN is defined as,

$$
M S E=\frac{1}{M X N} \sum_{i=0}^{M-1} \sum_{j=0}^{N-1}[O(i, j) \quad I(i, j)]^{2}
$$

where $I[\mathrm{i}, \mathrm{j}]$ is the noised original image and $O[\mathrm{i}, \mathrm{j}]$ is the denoised image.

Effective use of this method requires optimum values for several parameters such as maximum window size ' $\mathrm{W}_{\text {max }}$ ', threshold values $\mathrm{T}_{\mathrm{L}}$ and $\mathrm{T}_{\mathrm{H}}$ and the number of iterations. To maximize the probabilities that only wanted pixels take part in filtering operation and to reduce the computational time, above said parameters must be chosen of appropriate values. An optimal value for the maximum window size ' $\mathrm{W}_{\max }$ ' is chosen by running the proposed 
method on several standard test images. The maximum window size and the time needed for computation at different noise levels are shown in table (1). It has been observed that the window sizes needed for $10 \%$ to $90 \%$ noise density range between 3 to 9 . Table 2 shows the better performance of the proposed NAF over the filter schemes described in [12], [17], [18] and [22] for low to high noise density cases.

From the results it is seen that due to the usage the fixed value of empirically set threshold[12], and single pass execution $[12,17,18]$ the detection and restoration performances are low whereas the proposed method NAF uses an adaptive process of selecting the desired window size dynamically and try to avoid the participation of unwanted pixels, i.e. ' 0 ' and ' 255 ' in filtering operation and hence exhibits much better performance in terms of peak signal to noise ratio (PSNR) values. Effective use of this method requires a proper value of threshold, window size and iterations which maximize the probabilities that the only wanted pixels will take part in filtering operation and reduces the computational times. To choose an optimal value which can be used for all types of gray images, the proposed method has been tested on different standard test images. Table 1 shows the maximum window size reached, the computational time and PSNR results obtained at different noise levels for 'Lena', 'Baby ', and 'Leaf' images. In the proposed work, thus window size is automatically adapted based on the density of noise in the image as well as the density of corruption local to a window. The scheme is simple to implement and do not require large number of iterations. Table 2 presents the PSNR results obtained for the noisy 'Lena' image at noise densities ranging from $10 \%$ to $90 \%$. From the results it is evident that the proposed filter out performs all other filtering schemes that use fixed and empirically obtained threshold values and fixed size windows. Comparative analysis reveals that the proposed scheme has improved performance over other schemes [12], [17], [18], [20] preferably in medium-to-high density impulse noise cases. It is also observed that the computational overhead is less as compared to other proposed competent [19],[20] schemes.

The efficacy of the proposed scheme is also evaluated with respect to the perceptual results. Set of figures 1,2 and 3 presented below are the restoration results obtained for 'Lena' and 'Baby' and 'Leaf' images corrupted with 70\% noise density with the algorithms proposed [12],[17],[18] and [20] and the NMF. From the perceptual analysis, it is evident that the proposed algorithm performs with much better noise removal and detail preservation capability.

Table 1. Maximum Value of Window Size $\left(\mathrm{W}_{\max }\right)$ Attained, with 'Lena', 'Baby' and 'Leaf' Images for Noise Density (N.D) from $10 \%$ to $90 \%$.

\begin{tabular}{|c|c|c|c|}
\hline $\begin{array}{c}\text { \% } \\
\text { Noise } \\
\text { Density }\end{array}$ & \multicolumn{3}{|c|}{$\begin{array}{c}\text { Maximum Value of } \\
\text { Window Size Attained } \\
\mathbf{W}_{\max }\end{array}$} \\
\cline { 2 - 4 } & Lena & Baby & Leaf \\
\hline 10 & 3 & 3 & 3 \\
\hline 20 & 3 & 3 & 3 \\
\hline 30 & 3 & 3 & 3 \\
\hline 40 & 3 & 5 & 3 \\
\hline 50 & 5 & 5 & 3 \\
\hline 60 & 5 & 7 & 5 \\
\hline 70 & 7 & 9 & 5 \\
\hline 80 & 9 & 11 & 7 \\
\hline 90 & 11 & 11 & 7 \\
\hline
\end{tabular}


Table 2 . PSNR Results Obained for 'Lena', 'Baby' and 'Leaf' Images for Noise Density from $10 \%$ to $90 \%$.

\begin{tabular}{|c|c|c|c|}
\hline \multirow[t]{2}{*}{ \% Noise Density } & \multicolumn{2}{|c|}{ PSNR in } & dB \\
\hline & Lena & Baby & Leaf \\
\hline 10 & 44.9 & 44.4 & 44.5 \\
\hline 20 & 41.0 & 39.7 & 39.9 \\
\hline 30 & 38.1 & 37.9 & 38.0 \\
\hline 40 & 36.2 & 36.0 & 35.9 \\
\hline 50 & 34.4 & 34.3 & 34.1 \\
\hline 60 & 32.9 & 33.0 & 32.9 \\
\hline 70 & 31.5 & 31.2 & 31.0 \\
\hline 80 & 29.5 & 28.6 & 28.2 \\
\hline 90 & 27.2 & 26.2 & 25.9 \\
\hline
\end{tabular}

Table 3. Results of Execution Time obtained for 'Lena', 'Baby' and 'Leaf' Images for Noise Density from $10 \%$ to $90 \%$

\begin{tabular}{|c|c|c|c|}
\hline \multirow{2}{*}{$\begin{array}{c}\text { \% Noise } \\
\text { Density }\end{array}$} & \multicolumn{3}{|c|}{ Execution Time T(exc) in } \\
Seconds \\
\cline { 2 - 4 } & Lena & Baby & Leaf \\
\hline 10 & 3.56 & 3.10 & 2.89 \\
\hline 20 & 5.18 & 4.98 & 4.40 \\
\hline 30 & 7.23 & 6.95 & 6.28 \\
\hline 40 & 10.2 & 9.32 & 8.94 \\
\hline 50 & 13.3 & 12.9 & 12.3 \\
\hline 60 & 18.7 & 17.4 & 16.9 \\
\hline 70 & 26.3 & 26.0 & 25.7 \\
\hline 80 & 40.2 & 39.9 & 38.2 \\
\hline 90 & 80.1 & 78.9 & 77.8 \\
\hline
\end{tabular}

Table(4). PSNR (dB) Results of Different Techniques for LENA Image Corrupted With Various Noise Densities. ('ND' is Noise Density)

\begin{tabular}{|c|c|c|c|c|c|}
\hline \% ND & A1 & A2 & A3 & A4 & NAF \\
\hline 10 & 43.10 & 40.97 & 40.2 & 40.23 & 44.98 \\
\hline 20 & 38.51 & 38.31 & 36.32 & 39.25 & 41.04 \\
\hline 30 & 36.67 & 35.12 & 33.4 & 28.42 & 38.13 \\
\hline 40 & 34.83 & 33.47 & 32.27 & 33.72 & 36.22 \\
\hline 50 & 33.25 & 32.01 & 30.54 & 32.10 & 34.45 \\
\hline 60 & 31.87 & 30.78 & 29.63 & 30.41 & 32.96 \\
\hline 70 & 30.37 & 29.41 & 28 & 26.42 & 31.57 \\
\hline 80 & 28 & 27.87 & 26.53 & 22.87 & 29.52 \\
\hline 90 & 25.83 & 25.62 & 24.2 & 18.21 & 27.24 \\
\hline
\end{tabular}

In the above table $\mathrm{A} 1, \mathrm{~A} 2, \mathrm{~A} 3$ and $\mathrm{A} 4$ are the proposed algorithms explained in References $[9],[16],[17]$ and [18] 

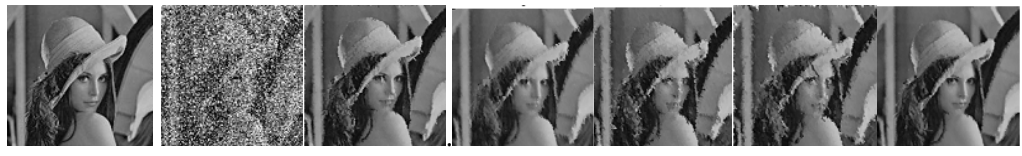

Figure 1. Results obtained for 'Lena' Image Corrupted with 70\% Noise Density
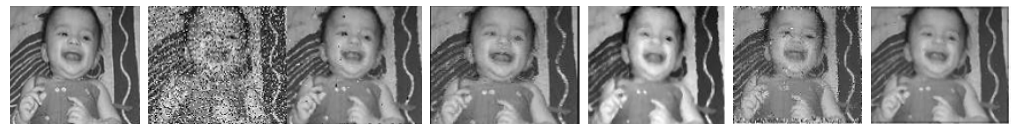

Figure 2. Results obtained for 'Baby' Image Corrupted with 70\% Noise Density
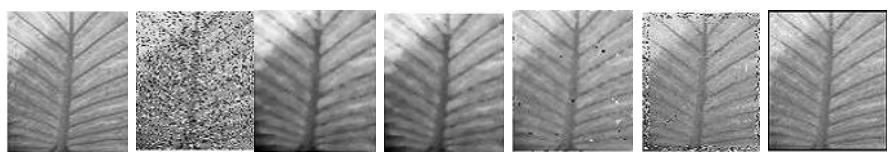

Figure 3. Results obtained for 'Leaf' Image Corrupted with 70\% Noise Density

\begin{tabular}{|l|l|l|l|l|l|l|}
\hline $\mathrm{a}$ & $\mathrm{b}$ & $\mathrm{c}$ & $\mathrm{d}$ & $\mathrm{e}$ & $\mathrm{f}$ & $\mathrm{g}$ \\
\hline
\end{tabular}

(a)Original (b) Noisy (c) Restored with A1, Ref [12] (d) Restored with A2 . Ref [17]

(e) Restored with A3, Ref [18] (f) Restored with A4 , Ref [20] (g) Restored with Proposed $\mathrm{NAF}$

\section{CONCLUSION AND FUTURE SCOPE}

An efficient algorithm that suppresses impulsive noise from an image heavily damaged by impulsive noise, without adding edge distortions is presented in this paper. Proposed Novel Adaptive (NAF) is Noise Exclusive Median based Filter and exhibits better performance in comparison with the filter algorithms [9], [16 ],[17] and [ 18] and several other recently proposed and improved noise removal algorithms in terms of PSNR, MSE and the perceptual results. The performance of the proposed scheme has been tested at low, medium and high noise densities. From the simulation results it is evident that the proposed filter scheme serves as a very good choice for salt -and-pepper noise suppression with detail preservation in noisy images. However, the major issue in this algorithm is computational complexity. The proposed filter scheme is designed for 8 bit images. The method can be enhanced for noisy image sequences, color images and super resolution images.

\section{ACKNOELEDGEMENTS}

Thanks to the reviewers for providing the feedback to improve the quality of the manuscript, especially with respect to the citation of the references. 
International Journal on Bioinformatics \& Biosciences (IJBB) Vol.4, No.3/4, December 2014

\section{REFERENCES}

[1] I.A.Pitas and A.N. Venetsanopolous, "Nonlinear digital filters principles and applications," Kluwer Press, Dordrecht, 1990.

[2] S.E.Umbaugh, "Computer Vision and Image Processing, "Prentice-Hall, Englewood Cliffs,NJ, USA, 1998.

[3] E. R. Dougherty, and J. Astola, eds., Nonlinear Filtering for Image Processing, SPIE and IEEE Presses, Belingham, 1999.

[4] O.Yli-Harja,J. Astola, and Y.Neuvo,"Analysis of the properties of median and weighted median filters using threshold logic and stack filter representation," IEEE Trans. Signal Processing , vol. 39, no. 2, pp. 395-410,1991.

[4] R. C.Gonzalez, and Woods R.E, "Digital Image Processing," Addison-Wesley, Boston, 2002.

[5] Wei-Yu Han and Ja-Chen Lin, "Minimum-maximum exclusive mean (MMEM) filter to remove impulse noise from highly corrupted images," Electronics Letters, vol.33, no.2,16th January, 1997.

[6] Z.Wang and D. Zhang, "Progressive switching median filter for the removal of impulse noise from highly corrupted images, "IEEE Trans. on Circuits and Systems II: Analog and Digital Signal Processing, Vol. 46,no. 1, pp. 78-80, 1999.

[7] ChenT.et al, "Tristate median filter for image denoising”, IEEE Transactions on Image Processing, Vol.8,No.12,pp.1834-183,1999.

[8] How-Lung Eng and Kai-Kuang Ma, "Noise adaptive soft-switching median filter (NASSMF),"IEEE Transactions on Image Processing” Vol. 10, no.2, pp. 242-251,2001.

[9] S.Esakkirajan, T.Veerakumar, Adabala N. Subramanyam and C. H. PremChand, "Removal of High Density Salt and Pepper Noise Through Modified Decision Based Unsymmetric Trimmed Median Filterl”, IEEE Signal Processing Letters, Vol. 18, NO.5, May 2011.

[10] Madhu N.S.,Revathy K., Tatavarti.R.,"An Improved Decision Based Algorithm (IDBA) for Impulse Noise Removal," Proceedings of 2008 International Congress on Image and Signal ProcessingCISP 2008, pp.426-431,IEEE Computer Society, 2008.

[11] Srinivasan, K.S., Ebenezer.D.,"A new fast and efficient decision based algorithm (FSDBA) for removal of High-density impulse noise," IEEE Signal Processing Letters, Vol.14, no. 3, pp. 189-192, 2007.

[12] Geeta Hanji, M.V.Latte,"A New Threshold Based Median Filtering Technique for Salt and Pepper Noise Removal," $2^{\text {nd }}$ International Conference on Digital Image Processing-(ICDIP-2010), 2010 Proceedings of SPIE, Vol.7546,PP754639-754639-10,SINGAPUR,2010,DOI: 10.1117/12.856333.

[13]C.Wang,T.Chen and Z.Qu,"A Novel Improved Median Filter for Salt-and-Pepper Noise from Highly Corrupted Images," $3^{\text {rd }}$ International Symposium on Systems and Control in Aeronautics and Astronautics (ISSCAA 2010),Harbin,China, pp.718-722, IEEE, 8-10 June,2010.

[14] Geoffrine Judith.M.C,N.Kumarasabapathy,"Study and analysis of impulse noise reduction filters", Signal \& Image Processing : An International Journal(SIPIJ),Vol.2, No.1, March 2011,DOI : 10.5121/sipij.2011.210782

[15] Mohammaed Ghouse,M.Siddappa,"Adaptive techniques based high impulsive noise detection of digital images", Journal of Theoretical and Applied Information Technology@2005 - 2011 JATIT \& LLS. www.jatit.org

[16]T. Ravi Kishore,K. Deergha Rao, "Efficient Median Filter for Restoration of Image and Video Sequences Corrupted by Impulsive" IETE Journal of Research,Volume 56, Issue 4, DOI:10.4103/0377-2063.70645,pages 219-226 Published online: 01 Sept 2014.

[17] Geeta Hanji, M.V.Latte, "Detail Preserving Fast Median Based Filter," Journal of Advanced Computer Science and Technology,1(4) (2012) 195-206 (C) Science Publishing Corporation, www.sciencepubco.com/index.php/JACST article/view/248

[18] Geeta Hanji,M.V.Latte, "A New Impulse Noise Detection and Filtering Algorithm," Image Processing \& Communications (IPC), The Journal of University of Technology and Life Sciences in Bydgoszcz. Vol. 16,no.1-2, pp.43-48,DOI: 10.2478/v10248-012-0004-4

[19]Ng PE, Ma KK., "A switching median filter with boundary discriminative noise detection for extremely corrupted images", IEEE Transactions on Image Processing, 1.15(.6): 1506-1516,2006.

[20] A.K. Tripathi, U. Ghanekar and S.Mukhopadhyay, "Switching median filter: Advanced boundary discriminative noise detection algorithm," IET Image Processing., Vol 5, issue 7, pp 598-610, 2011. 
[21]Dr. G. Venkata Rami Reddy, Dr. B. Sujatha, "Directional Correlation-Dependent FilteringTechnique for Removal of Impulse Noise",International Journal of Signal Processing, Image Processing and Pattern Recognition,Vol.7, No.4 (2014), pp.85-92,http://dx.doi.org/10.14257/ijsip.2014.7.4.08.

[22] Geeta Hanji,M.V.Latte, "Novel Median Filter for Impulse Noise Suppression from Digital Images," International Journal of Computer Applications (IJCA), (0975 - 8887), Volume 106- No.8, November 2014.

\section{Authors}

Geeta Hanji is currently working as Associate Professor in the department of E \& CE,PDA Engineering College,Gulbarga,Karnataka,India.She received BE degree from Mysore University in the year 1990 and M.Tech degree in the year 2002 from Vishveswaraya Technological University, Karnataka, India. She is research scholar of JNTU,Hyderabad. Her research interests are Image Processing, Wireless Networks and Data Networks.

Dr.M.V.Latte is currently working as Principal, JSSIT, Bangalore, Karnataka, India. He received B.E.,M.E. and Ph.D. degrees from Karnataka University, Dharwad, Karnataka, India. His research interests are Signal Processing, Image Processing and Digital Communication. 